\title{
Social Reminiscence in Older Adults' Everyday Conversations: Automated Detection Using Natural Language Processing and Machine Learning
} \author{
Prof Dr

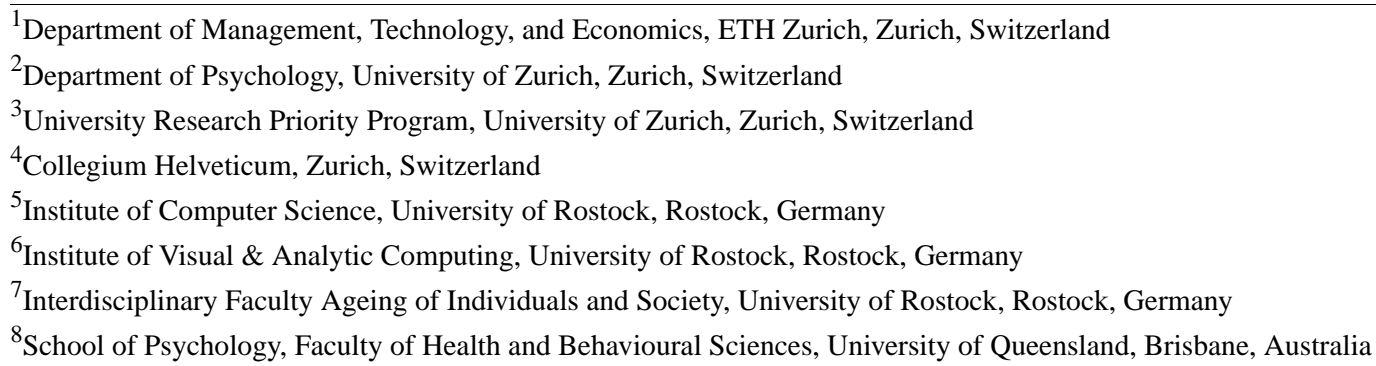

Andrea Ferrario $^{1}$, PhD; Burcu Demiray ${ }^{2,3,4}, \mathrm{PhD}$; Kristina Yordanova ${ }^{5,6,7}$, Dr; Minxia Luo ${ }^{2,3}$, MSSc; $_{\text {Mike Martin }}^{2,3,4,8}$,

Corresponding Author:

Andrea Ferrario, $\mathrm{PhD}$

Department of Management, Technology, and Economics

ETH Zurich

Weinbergstrasse $56 / 58$

Zurich, 8092

Switzerland

Phone: 41446328624

Email: aferrario@ethz.ch

\section{Abstract}

Background: Reminiscence is the act of thinking or talking about personal experiences that occurred in the past. It is a central task of old age that is essential for healthy aging, and it serves multiple functions, such as decision-making and introspection, transmitting life lessons, and bonding with others. The study of social reminiscence behavior in everyday life can be used to generate data and detect reminiscence from general conversations.

Objective: The aims of this original paper are to (1) preprocess coded transcripts of conversations in German of older adults with natural language processing (NLP), and (2) implement and evaluate learning strategies using different NLP features and machine learning algorithms to detect reminiscence in a corpus of transcripts.

Methods: The methods in this study comprise (1) collecting and coding of transcripts of older adults' conversations in German, (2) preprocessing transcripts to generate NLP features (bag-of-words models, part-of-speech tags, pretrained German word embeddings), and (3) training machine learning models to detect reminiscence using random forests, support vector machines, and adaptive and extreme gradient boosting algorithms. The data set comprises 2214 transcripts, including 109 transcripts with reminiscence. Due to class imbalance in the data, we introduced three learning strategies: (1) class-weighted learning, (2) a meta-classifier consisting of a voting ensemble, and (3) data augmentation with the Synthetic Minority Oversampling Technique (SMOTE) algorithm. For each learning strategy, we performed cross-validation on a random sample of the training data set of transcripts. We computed the area under the curve (AUC), the average precision (AP), precision, recall, as well as F1 score and specificity measures on the test data, for all combinations of NLP features, algorithms, and learning strategies.

Results: Class-weighted support vector machines on bag-of-words features outperformed all other classifiers (AUC $=0.91$, $\mathrm{AP}=0.56$, precision $=0.5$, recall $=0.45, \mathrm{~F} 1=0.48$, specificity $=0.98)$, followed by support vector machines on $\mathrm{SMOTE}$-augmented data and word embeddings features $(\mathrm{AUC}=0.89, \mathrm{AP}=0.54$, precision $=0.35$, recall $=0.59, \mathrm{~F} 1=0.44$, specificity $=0.94$ ). For the meta-classifier strategy, adaptive and extreme gradient boosting algorithms trained on word embeddings and bag-of-words outperformed all other classifiers and NLP features; however, the performance of the meta-classifier learning strategy was lower compared to other strategies, with highly imbalanced precision-recall trade-offs. 
Conclusions: This study provides evidence of the applicability of NLP and machine learning pipelines for the automated detection of reminiscence in older adults' everyday conversations in German. The methods and findings of this study could be relevant for designing unobtrusive computer systems for the real-time detection of social reminiscence in the everyday life of older adults and classifying their functions. With further improvements, these systems could be deployed in health interventions aimed at improving older adults' well-being by promoting self-reflection and suggesting coping strategies to be used in the case of dysfunctional reminiscence cases, which can undermine physical and mental health.

(J Med Internet Res 2020;22(9):e19133) doi: 10.2196/19133

\section{KEYWORDS}

aging; dementia; reminiscence; real-life conversations; electronically activated recorder (EAR); natural language processing; machine learning; imbalanced learning

\section{Introduction}

\section{Reminiscence and Healthy Aging}

The world's population is rapidly aging. With its first world report on aging and health [1], the World Health Organization (WHO) promoted a global paradigm shift in aging research by moving from a disease-focused model to a dynamic, contextualized, person-focused model of "healthy aging" [1]. This model emphasizes the interplay of personal characteristics (eg, abilities), environments, and their interactions in producing functioning. Activities represent the interaction between person characteristics and environments; they are understudied in traditional aging research. The novel WHO model encourages aging researchers to step outside the lab and into the real world to examine activities in everyday life [2], aiming to empower individuals to observe, measure, and take earlier action for their own health $[3,4]$. In this study, we embrace the healthy aging model by examining one such real-life activity: reminiscence.

Reminiscence [5] is the "naturally occurring act of thinking about or telling others about personally meaningful past experiences" [6,7]. These experiences may refer to specific events (eg, the first kiss), repeated ones (eg, going to the gym every Friday), extended ones (eg, a Christmas trip), or even long periods of life (eg, living in a foreign country for some years) [8]. Recalling or sharing valuable life experiences with third parties can support decision-making, bonding with others, and self-understanding [9]. Reminiscing can be a volitional or nonvolitional process recollecting memories [7], an activity that may be private or involve others [7]; in the latter case, we refer to social reminiscence. Many disciplines are interested in the study of reminiscence, such as nursing, social work, education, theology, psychology, and gerontology [10], with a strong focus on reminiscence in the context of aging. Researchers who study aging emphasize a cognitive activity in old age such as reminiscence to be an essential part of healthy aging [11]; in fact, the use of memory interventions and reminiscence in therapies for older adults is common, emphasizing the relation between self-positive functions of reminiscence and well-being [12], according to Webster and Cappeliez's tripartite model of reminiscence [13].

\section{Naturalistic Observation as a New Approach to the Study of Reminiscence}

The study of reminiscence in older adults has traditionally focused on (1) reflective self-reporting and life reviews [11] and (2) automated reminiscence therapy, that is, "a nonpharmacological intervention involving the prompting of past memories, [...] for therapeutic benefits, such as the facilitation of social interactions or the increase of self-esteem" [14], especially for dementia patients.

The use of self-reporting has potential limitations, such as recall biases, response styles, demand characteristics, social desirability, and limitations to introspection [15]. Moreover, the self-report method provides researchers only with the average frequency of an activity over a certain period of time [16]. Studies with a focus on automated reminiscence therapy, in contrast, typically aim at eliciting reminiscence from users (eg, with the remote assistance of a therapist), rather than collecting spontaneous reminiscence events during everyday life settings [17-19].

In 2017, Demiray et al [6] were the first to examine reminiscence using a naturalistic observation method to enable investigating reminiscence in the real world. As opposed to those used in the reminiscence therapies, this method does not rely on self-report and tracks objective behaviors, such as speech in everyday life, with no elicitation of reminiscence events. It can also involve older adults in scientific investigations who would otherwise be excluded from real-life studies relying on self-reporting (eg, older adults who are intimidated by technology or therapy are unable to use a smartphone to complete surveys or to self-report due to worsened eyesight, and/or are part of a clinical population). Furthermore, this method allows for microlongitudinal study designs with many measurement points per participant and both within- and between-persons perspectives in analyses.

However, to understand what kinds of reminiscence patterns are predictive of maintaining healthy aging and quality of life [2], each instance of reminiscing has to be reliably detected in everyday life contexts. In fact, reminiscing is a context-dependent, real-world cognitive activity [20]; if it can be reliably and accurately assessed, it is possible to study the effects of real-world cognitive activities on health outcomes, such as cognitive abilities or cognitive impairments [6,12]. A rich body of literature exists on the link between reminiscence therapies and cognitive and well-being benefits in aging populations: a recent review [21] based on 22 randomized controlled studies showed evidence for the positive effects of reminiscence therapy on quality of life, cognition, and communication in dementia patients. Subramaniam and Woods [22] specifically reviewed the effects of individual-based therapy 
and concluded that it shows immediate benefits on well-being and cognition. The challenge, however, is to find automated ways to extract and disambiguate the cognitive activity information from data streams collected from many persons in real-world situations [23]. Tracking, detecting, and prompting functional as well as positive reminiscence behaviors in everyday life should allow researchers to design digital interventions to enhance the quality of life of healthy older adults and other patient populations. To these ends, natural language processing (NLP) and machine learning methodologies allow researchers to explore the possibility of reliably predicting reminiscence in combination with naturalistic observation methods and in real time.

\section{Using NLP and Machine Learning for Reminiscence}

Yordanova et al [24] were the first to investigate the applicability of NLP and machine learning methodologies on data from a naturalistic observation study by Demiray et al [6]; they introduced an NLP pipeline and machine learning routines to automatically code the social behaviors and interactions (eg, talking to a partner or daughter/son, giving advice, receive support, etc) in the transcripts of recorded conversations. As coding is a manual process that involves much effort and time, their results showed that the use of NLP and machine learning automation on transcripts of recorded conversations enabled reliable coding of social behaviors and interactions, reducing effort and time. However, they did not consider detecting reminiscence. Their proposed pipeline included data augmentation procedure to cope with highly imbalanced classes, feature engineering based on linguistic, contextual and statistical approaches, and supervised learning with classifiers such as decision trees, random forests (RF), and support vector machines (SVM). As the extreme imbalance between classes poses a central problem in automated coding of textual data, other works propose training classifiers with annotated data sets and later performing manual evaluation to correct misclassified instances by experts [25]. This second manual step is expensive and time-consuming, but it shows that NLP and machine learning deliver promising results with respect to the automated analysis of textual data in social science applications. In fact, the use of NLP and machine learning methods has shown great potential for analyzing social media posts for social affect and behavior, identifying trends in society and demographics, as well as generating predictions of society-changing events, such as diseases [26-28].

Motivated by the availability of reminiscence data from the naturalistic observation study [6] and the results of Yordanova et al [24], this study aims to develop pipelines with NLP and machine learning strategies to automatically detect reminiscence in older adults' everyday conversations in German using their written transcripts. To do so, we introduce various NLP features (bag-of-words models, part-of-speech [POS] tagging, and pretrained word embeddings) to preprocess written transcripts, which are fed into four families of machine learning algorithms (RF, adaptive boosting [ADA], extreme gradient boosting [XGB], and SVM); multiple learning strategies are setup to cope with class imbalance in data. The methods of this study support the understanding of a key activity of the healthy aging model, that is, reminiscence, in a real-world setting by leveraging transcriptions of everyday life conversations. Moreover, they could support the design of computer systems to detect social reminiscence in the everyday lives of older adults in real time and classify different reminiscence functions. These systems lie at the core of digital health intervention programs [29] aimed at improving older adults' well-being by promoting self-reflection, as suggested by the healthy aging model. They also provide users with coping strategies for dysfunctional reminiscence [13], which has a negative emotional valence and affects physical and mental health [30-32]. Even when the real-time recording of older adults' memory activities contained in daily conversations in a research study is shown to be feasible, there are multiple challenges to scaling up this intervention to a large population. However, current findings suggest that despite potential concerns about privacy and data protection issues, there are now a number of technical [33] and analytical solutions for privacy-preserving machine learning for such data [34]. Additionally, a majority of older adults is willing to share portable data collections with researchers [3].

\section{Methods}

\section{Overview of the Study Design}

This study comprises the following steps: (1) collecting data from a naturalistic observation study [6], (2) preprocessing data with NLP methodologies, and (3) training and validating machine learning models to detect reminiscence in a given corpus of transcriptions, by implementing three distinct learning strategies to cope with class imbalance in the data.

\section{Data Collection: Older Adults' Everyday Conversations and Reminiscence}

\section{Data Source}

The aim of the naturalistic observation study by Demiray et al [6] was to collect everyday conversations of older adults and examine social reminiscence behavior. Random snippets of older adults' everyday conversations were collected using the Electronically Activated Recorder (EAR) [35]. They generated 13,275 audio files from 48 older adults ( 22 men and 26 women) residing in Zurich, Switzerland, over a period of 4 days. The average age of the sample was 70.54 years (SD 4.65, range 62-83 years). To be eligible for the study, participants were required to have a minimum score of 27 on the Mini Mental State Examination [36]. The participants had an average of 10.5 years of education (SD 3.0, range 7-25 years), and they all spoke Swiss German. The study included an introductory session, an observation period, and a feedback session. In the introductory session of the study, participants signed informed consent and received an iPhone in which the EAR was installed. They were informed that the EAR would randomly record a few seconds of audio multiple times per day, except for an automatically inactivated period from every midnight to $6 \mathrm{AM}$ the next day, during the whole observation period. They were told to carry the iPhone and continue daily living, and they were informed that they would not notice when the EAR was recording.

The observation period started the day after the introductory session and lasted 4 consecutive days, during which the audio file recording occurred. After the observation period, the 
participants were invited to the feedback session, where they returned the phones, completed further questionnaires, and provided their feedback about their experiences of carrying the EAR. They received password-protected CDs containing all of their audio files. All study procedures were approved by the Ethics Research Institute of the Department of Philosophy at the University of Zurich.

\section{The EAR App}

The EAR (version 2.3.0) [35,37] was installed on each iPhone. It was set to randomly record 30-second audio files 72 times over 4 days. Thus, each participant was recorded 288 times and for a total 144 minutes each. Each iPhone was set to "airplane mode" and locked with a screen-lock password. The participants were instructed to charge the iPhone in the evenings. At the end of the study, the participants reported that the EAR did not affect their daily activities or way of speaking [20].

\section{Data Generation: Transcribing and Coding Audio Files}

For each audio file, all utterances by the study participants were transcribed by two research assistants, who were fluent in Swiss German and standard written German. Swiss German is an Alemannic dialect spoken in the German-speaking part of Switzerland, which does not have a standard written form. Thus, the Swiss-German dialect in the audio files was translated word-by-word into standard written German and then transcribed. Coders generated binary variables (with values 0 and 1), indicating whether the participant was talking or not and whether he or she was reminiscing or not. The function(s) of reminiscence [8] and participants' conversation partners (ie, partner/spouse, daughter/son, other family members, etc) were also coded.

\section{Coding Reminiscence}

Two coders performed the manual coding of reminiscence in each audio file independently. The interrater reliability for the coding of reminiscence, that is, the percentage of audio files with the same coding assigned by both coders, was $95 \%$. All discrepancies in coding were solved by relistening and recoding through discussion. To classify reminiscence, the coders generated a binary variable with values 0 and 1 , which correspond to a general conversation and a reminiscence case, respectively.

\section{Preparing Data}

Of the 13,275 audio files generated during the study by Demiray et al [6], 2214 contained conversations of older adults. The data set used for this study comprises these 2214 transcripts, of which $109(4.9 \%)$ were coded as reminiscence. Before applying NLP to the transcripts, we preprocessed the data with regular expressions to remove coding artifacts like "xxx," "YYY," "xxxx," etc, denoting utterances from the audio recording that were not possible to transcribe as well as leading and trailing whitespaces.

\section{Natural Language Processing of Transcripts}

To use written transcripts as inputs of machine learning models, we preprocessed them by computing the NLP features (1) "bag-of-" models on both words and POS tags and (2) real-valued embeddings for each transcript in the data set using pretrained German word embeddings.

\section{Bag-of-Words Models}

Bag-of-words models [38,39] represent transcripts as real-valued vectors by tokenizing all transcripts in the provided data set, collecting unique tokens, and counting their occurrences before applying normalization (eg, term frequency-inverse document frequency [tf-idf] [38,39]). Bag-of-words models do not consider the order of words in transcripts and generate high-dimensional representation of textual data. In fact, bag-of-words models represent each transcript by a real-valued vector whose dimension is equal to the size of the vocabulary of the whole data set of transcripts. They are widely used for text classification tasks, including studies in digital health [29,40-42]. We computed bag-of-words models using the TfIdfVectorizer() function in the Python sklearn library [43].

\section{Bag-of-POS Models}

POS tagging is the process of assigning a POS tag to each word in a given corpus [38,39]; the algorithm that performs the tagging is called a POS tagger; a set of all tags is called a tagset. POS tagging enables including information from a word's context (ie, its relationships with close and related words in a document) in text classification tasks [29]. In this study, we used the POS tagger provided in the core model for the German language "de_core_news_sm," which is available in the Python library SpaCy [44], to generate the POS tags for all tokens retrieved from the corpus of 2214 transcripts. The SpaCy POS tagger has a tagset comprising 17 distinct tags; similar to bag-of-words models, a bag-of-POS model extracts all POS tags (instead of words) from a transcript and counts their occurrences before applying normalization, such as tf-idf. With bag-of-POS models, one can encode information on the linguistic structure of each transcript in a real-valued, low-dimension representation.

\section{Word Embeddings}

Word embeddings are real-valued representations of textual data encoding the "distributional hypothesis" [45] about language and words: words that occur in similar contexts tend to be closer to each other as real-valued vectors. Moreover, word embeddings are generally real-vector representations of textual data of much lower dimension than, for example, those in bag-of-words models. They have emerged as a common technique to compute representations of textual data, including studies in digital health $[29,40]$. In this study, given the limited number of available transcripts, we opted for pretrained German word embeddings using the SpaCy core model for the German language "de_core_news_sm." The model is "German multi-task CNN trained on the TIGER and WikiNER corpus" [46] and each word embedding has 300 dimensions. The TIGER corpus [47] is curated by the Institute for Natural Language Processing at the University of Stuttgart; it comprises 900,000 tokens from sentences of German text, taken from the Frankfurter Rundschau newspaper [48]. On the other hand, WikiNER [49] is a corpus for multilingual named entity recognition from Wikipedia. In this study, the embedding of each transcript is a real vector of 
300 dimensions resulting from averaging the embeddings of all words generated from the given transcript after its tokenization.

\section{Machine Learning on Transcripts: Learning Strategies}

\section{Class Imbalance}

The data set of 2214 transcripts has 109 records coded as reminiscence; therefore, it has a class imbalance with a ratio of 20:1. A class imbalance [50-53] is a common phenomenon in machine learning and, in particular, in textual data [24,40,54,55]. Since most machine learning algorithms are biased toward the majority class [53], researchers have proposed various solutions to cope with learning with imbalanced data sets [50,51], such as class-based weighting of misclassification errors or data resampling techniques aimed at reducing imbalance [53]. Research has also promoted the use of performance measures, which consider the presence of class imbalance [53] to evaluate machine learning models.

\section{Machine Learning Methods}

In this study, to cope with class imbalance during the training of machine learning models to detect reminiscence, we implemented the following learning strategies: (1) class-weighted learning (CWL), (2) meta-classifier (MC) learning, and (3) learning in the presence of data augmentation with the Synthetic Minority Oversampling Technique (SMOTE) algorithm [56]. For all the learning strategies, we trained machine learning classifiers [57] using the RF, ADA, XGB [58], and SVM algorithms fed on all NLP features: bag-of-words, bag-of-POS NLP models, and pretrained word embeddings. In the cases of RF or boosting algorithms trained on either bag-of-words or bag-of-POS features, we computed feature importance using the sklearn property .feature_importances_. Table 1 summarizes all the NLP features, classifiers, and learning strategies used in this study.

Table 1. Summary of all natural language processing (NLP) features, machine learning algorithms (ie, classifiers), and learning strategies considered in this study.

\begin{tabular}{|c|c|}
\hline Features, algorithms, and strategies & Cases \\
\hline NLP features & $\begin{array}{ll}\text { - } & \text { Bag-of-words } \\
\text { - } & \text { Bag-of-POS } \\
\text { - } & \text { Pretrained German word embeddings }\end{array}$ \\
\hline Algorithm (classifier) & $\begin{array}{ll}\text { - } & \text { Random forests } \\
\text { - } & \text { Adaptive boosting } \\
\text { - } & \text { Extreme gradient boosting } \\
\text { Support vector machines }\end{array}$ \\
\hline Learning strategy & $\begin{array}{ll}\text { - } & \text { Class-weighted training } \\
\text { - } & \text { Meta-classifier training } \\
\text { - } & \text { Data augmentation with SMOTE }\end{array}$ \\
\hline
\end{tabular}

${ }^{\mathrm{a}} \mathrm{POS}$ : part-of-speech.

${ }^{\mathrm{b}}$ SMOTE: Synthetic Minority Oversampling Technique.

\section{Evaluation Metrics}

For all the learning strategies, the performance of the machine learning models on test data was computed using the area under curve (AUC), precision, recall (or sensitivity), the average precision (AP), specificity, and the F1 score measures. The AUC summarizes in a single number the performance of the classifier shown in the receiver operating curve $[59,60]$, which plots the true positive rate versus the false positive rate, at various classifier thresholds settings. Precision is the number of true positives (ie, transcripts containing reminiscence, which are correctly predicted by the machine learning model) divided by the number of transcripts, which are predicted to contain reminiscence by the model. Recall (or sensitivity) is the number of true positives divided by the number of transcripts containing reminiscence. The sklearn implementation of the AP [61] summarizes the precision-recall curve [62] as the weighted mean of precisions achieved at each threshold by the classifier, the increase in recall from the previous threshold used as the weighting. We chose to report the AUC and AP, as they provide a global overview of the classifier performance, for all possible classification thresholds. Specificity is the number of true negatives divided by the total number of negative instances, while the F1 score is the harmonic mean of precision and recall measures [38]; it is a common evaluation metric in the presence of imbalanced data.

The formulas for AP and F1 score are as follows:

$$
\begin{aligned}
& A P=\sum_{n}\left(R_{n}-R_{n-1}\right) P_{n} \\
& F 1=2 \text { precision } * \text { recall } / \text { precision }+ \text { recall })
\end{aligned}
$$

In the AP formula, $R_{n}$ denotes the recall computed at step $n$ (similarly for $R_{n-1}$ ), while $P_{n}$ is the precision at step $n$.

\section{Error Analysis}

To identify errors in detecting reminiscence, we analyzed the false positives with the highest predicted probabilities (10\% of all cases) and the false negatives with the lowest predicted probabilities (10\% of all cases) computed for all the models presented in Table 2. 


\section{Experimental Setting}

\section{Class-Weighted Learning}

We show the CWL strategy in Figure 1, top panel. After an initial partition of the data into train and test subsets with a 80:20 ratio, a 5-fold cross-validation routine was applied to the 1771 training data (87 reminiscence) to select the best model for all NLP features and families of classifiers shown in Table 1. We use the AUC to measure performance on the validation folds. The CWL strategy does not modify the imbalanced class distribution of training data, but reweighs them according to their class during the training of the machine learning algorithm at hand $[40,52,54]$, penalizing the cost of misclassifying data points from the minority class [52,63,64]. For example, for the RF, ADA, and SVM algorithms, we selected the parameter "class_weight=balanced" [65] in their sklearn implementations. Similar considerations held for XGB algorithms, where we selected "scale_pos_weight=weight," where weight denoted the ratio of the number of negative class samples to the positive class (ie, reminiscence) [66]. The best model resulting from the cross-validation was evaluated on test data, by computing AUC, AP, and F1.

Figure 1. Top panel: Class-weighted learning and data augmentation learning strategies. We performed a single data partition into train and test sets; the best classifier emerged from 5-fold cross-validation (CV). Bottom panel: meta-classifier learning strategy; we undersampled the training data 50 times, collecting 50 distinct models in a voting ensemble after CV. We applied the three strategies for all the combinations of natural language processing features and machine learning algorithms shown in Table 1.

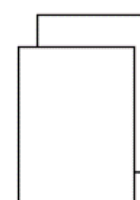

Data: transcriptions + coding
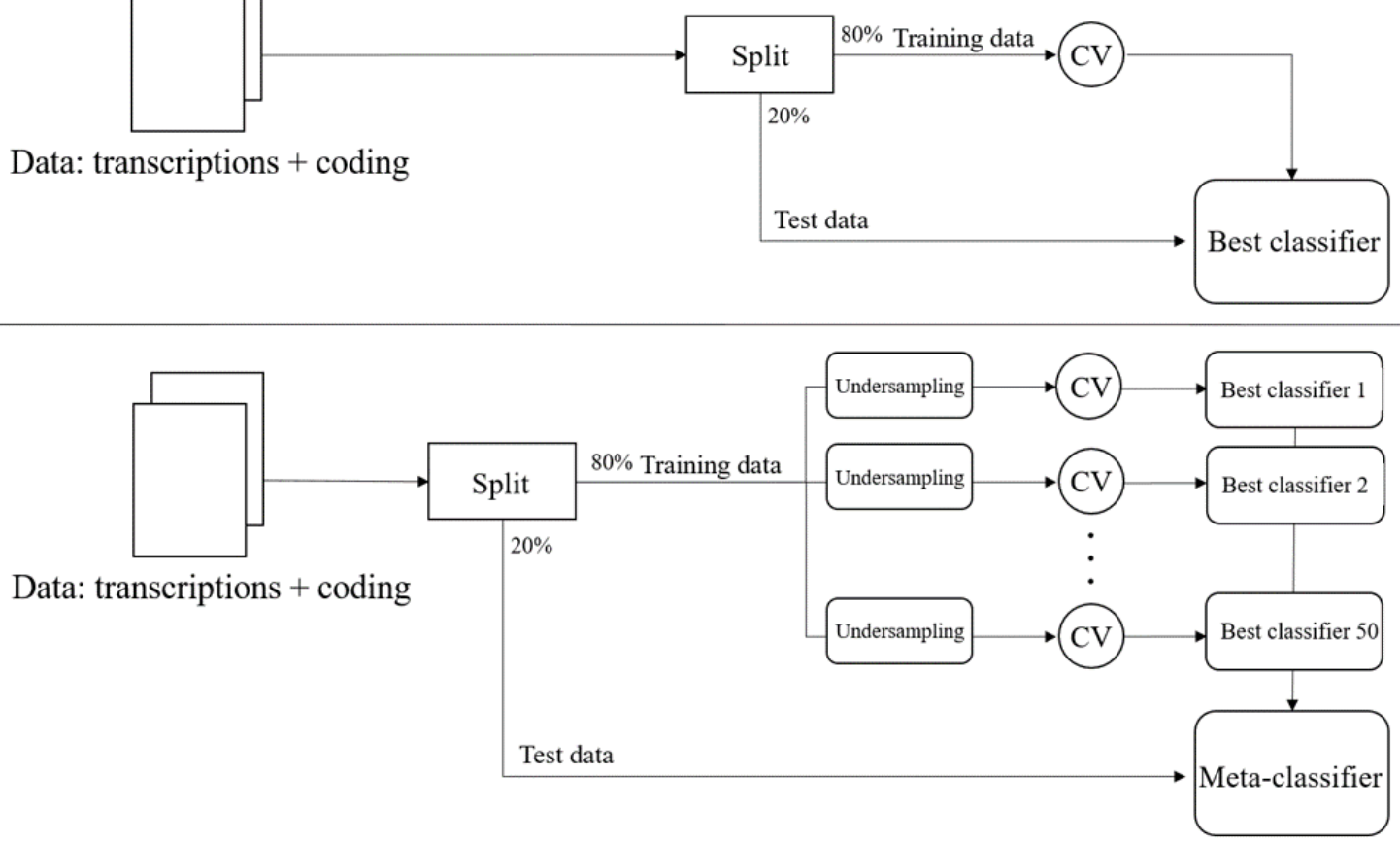

\section{Meta-Classifier Training}

We show the MC strategy in Figure 1 (bottom panel). The MC is a majority voting ensemble classifier [67] comprising 50 equally weighted distinct models resulting from 50 runs of 5-fold cross-validation on 50 randomly undersampled (with 1:1 ratio) training data sets. Undersampling is a common technique in imbalanced learning [41,68]; the use of voting allows reducing the bias of a single undersampling of training data. For each run, the cross-validation routine was performed on 174 training transcripts ( 87 reminiscence). The AUC and AP of the MC were computed on test data.

\section{Data Augmentation With SMOTE}

Figure 1 (top panel) shows the strategy involving data augmentation with the SMOTE algorithm: it follows the same steps as the one implemented for CWL. However, during the 5-fold cross-validation, the results of all NLP pipelines on transcripts are preprocessed with SMOTE before being fed into the machine learning classifiers. SMOTE [50] is an algorithm that generates synthetic examples of the minority class (in this study, the reminiscence class) in imbalanced data sets; given a minority class data point, SMOTE generates synthetic examples along line segments connecting the given data point to its $\mathrm{K}$ nearest neighbors ( $K=5$ is the default value). SMOTE is widely used to perform data augmentation in the presence of imbalanced learning, including clinical studies $[40,69,70]$. The Python implementation of the SMOTE algorithm also allows controlling the oversampling quota, that is, the ratio between the minority class (after resampling) and the majority class. For example, using SMOTE on training data to reach a 1:1 ratio among classes, we ran the cross-validation routines in Figure 1 (top panel) on a total of 3368 data points (1684 reminiscence).

\section{Cross-Validation}

For all three learning strategies, as shown in Figure 1, we performed cross-validation by tuning the hyperparameters of (1) the NLP pipelines computing bag-of-words and bag-of-POS features, together with those of (2) the machine learning 
algorithms before retrieving the best classifier (MC in the case of the voting learning strategy).

\section{NLP Preprocessing Pipelines}

For the bag-of-words features, we tuned the n-grams, German stopword list (ie, its removal or not), minimum document frequency, maximum document frequency, and maximum number of feature hyperparameters. After preprocessing, in case of 1-grams we have 6596 tokens, and 38,347 in the case of 2-grams. The preprocessing of the POS features follows the one for bag-of-words, with the exception of the German stopword list; in case of 1-grams we obtained 16 tokens, and 270 in the case of 2-grams.

\section{Machine Learning Models: Hyperparameters}

For ADA classifiers, we tuned the number of estimators and the learning rate; for XGB, we tuned the number of estimators, the learning rate, and the maximum tree depth; and for RF, we tuned the number of trees in the ensemble, their depth, and the number of features considered at each tree split during training. In addition, when considering the data augmentation learning strategy, we tuned the SMOTE number of nearest neighbors and oversampling quota hyperparameters. The NLP preprocessing pipelines are the same for all machine learning models.

\section{Results}

\section{Machine Learning Modeling}

Multimedia Appendix 1 displays the results of the machine learning modeling, reporting the best performing classifier for all NLP features and learning strategies, together with its AUC,
$\mathrm{AP}$, precision, recall, $\mathrm{F} 1$, and specificity performance measures, which we computed on test data (443 transcripts, 22 reminiscence).

Table 2 displays the two best performing classifiers (considering the F1 score as performance measure) for each learning strategy. We choose two best models per learning strategy to show how different combinations of machine learning algorithms and NLP features may result in different precision-recall trade-offs.

Considering the class weighting strategy, SVM outperforms all other classifiers, with the highest F1 score (0.48) when trained on bag-of-words features, with a balanced precision-recall trade-off and very high specificity (0.98). On the other hand, SVM trained on word embeddings shows higher AP (0.61) and recall (0.77); however, its lower precision (0.21) results in a lower F1 score (0.33), together with a lower specificity (0.85).

The best performing classifiers for the MC strategy, which are trained on word embeddings and bag-of-words features, show F1 scores ( 0.30 for both ADA and XGB classifiers) lower than those in the class-weighted and data augmentation strategies. This is due to low precision; similarly, they show low specificities. However, they reach very high recall (1.00 for ADA and 0.86 for XGB classifiers) and high AUCs.

Finally, for the data augmentation strategy, SVM on word embeddings shows highest F1 score (0.44), with recall (0.59) higher than precision (0.35) and high specificity (0.94). On the other hand, ADA classifiers trained on word embeddings show a more balanced precision-recall trade-off, with a higher precision (0.43) but lower recall (0.41) than the SVM classifier, resulting in a slightly lower F1 score $(0.42)$ but higher specificity (0.97).

Table 2. Summary of best models for each learning strategy, considering the F1 score.

\begin{tabular}{|c|c|c|c|c|c|c|c|}
\hline $\begin{array}{l}\text { Learning strategy and } \\
\text { NLP }^{a} \text { feature }\end{array}$ & Classifier family & $\mathrm{AUC}^{\mathrm{b}}$ & $\mathrm{AP}^{\mathrm{c}}$ & Precision & Recall & $\mathrm{F} 1$ & Specificity \\
\hline \multicolumn{8}{|l|}{ Class weighting } \\
\hline BOW $^{\mathrm{d}}$ & $\mathrm{SVM}^{\mathrm{e}}$ & 0.91 & 0.56 & 0.50 & 0.45 & 0.48 & 0.98 \\
\hline $\mathrm{EMB}^{\mathrm{f}}$ & SVM & 0.91 & 0.61 & 0.21 & 0.77 & 0.33 & 0.85 \\
\hline \multicolumn{8}{|l|}{ Meta-classifier } \\
\hline BOW & $\mathrm{XGB}^{\mathrm{g}}$ & 0.90 & 0.45 & 0.18 & 0.86 & 0.30 & 0.79 \\
\hline EMB & $\mathrm{ADA}^{\mathrm{h}}$ & 0.92 & 0.38 & 0.18 & 1.00 & 0.30 & 0.76 \\
\hline \multicolumn{8}{|l|}{ Data augmentation } \\
\hline EMB & SVM & 0.89 & 0.54 & 0.35 & 0.59 & 0.44 & 0.94 \\
\hline EMB & ADA & 0.84 & 0.36 & 0.43 & 0.41 & 0.42 & 0.97 \\
\hline
\end{tabular}

${ }^{\mathrm{a}} \mathrm{NLP}$ : natural language processing.

${ }^{\mathrm{b}} \mathrm{AUC}$ : area under the curve.

${ }^{\mathrm{c}} \mathrm{AP}$ : average precision.

${ }^{\mathrm{d}}$ BOW: bag-of-words.

e SVM: support vector machines.

${ }^{f}$ EMB: word embeddings.

${ }^{\mathrm{g}} \mathrm{XGB}$ : extreme gradient boosting.

${ }^{\mathrm{h}}$ ADA: adaptive boosting. 


\section{Feature Analysis}

Overall, considering Table 2 and the F1 score, the use of word embeddings outperformed other NLP features for the data augmentation strategy; it delivers performance equal to the one with bag-of-words features for the MC strategy; on the other hand, for class weighting, bag-of-words features deliver the highest performance with the SVM classifier.

Considering bag-of-words features, for the data augmentation strategy XGB outperformed the other classifiers (with 1-grams, and no German stopword removal) with 300 boosting iterations, shallow trees (ie, a depth of 1), and the number of neighbors used by SMOTE equal to $K=13$. The words with the highest feature importance were German stopwords (eg, "gewesen," "und," "wir," "ist," and "ich" ["been," "and," "we," "is," and "I"]). Training the same classifier but removing the stopwords led to a strong decline in performance $(\mathrm{AUC}=0.79, \mathrm{AP}=0.22$, F1 = 0.27); in this case, the words with the highest feature importance comprised adverbs (eg, "aber," and "einfach" ["but" and "simply"]) and past participles (eg, "gesagt" and "gehabt" ["said" and "had"]), among others.

Considering POS features and the class weighting strategy, XGB delivered the highest performance in the presence of $1-$ and 2-grams, with few boosting iterations (ie, 50) and shallow trees (ie, a depth of 1). The 1-grams with the highest feature importance were auxiliary verb forms (eg, imperative, infinitive, perfect participle of "sein," "haben," and "werden" [ie, "to be," "to have," and "to become"]), conjunctions, and adverbs, while the 2-grams comprised verbs and auxiliary verbs (eg, the combinations of a noun and the past participle of "sein," "haben," and "werden"), adverbs and verbs (eg, "aber weisst," "dann sagt," or "mehr gegessen”).

On the other hand, considering the data augmentation strategy and POS features, the XGB algorithm presented in Multimedia Appendix 1 performs on 10 boosting iterations, in the presence of shallow trees, 2-grams, and $\mathrm{K}=5$ neighbors used by SMOTE. In the case of the class weighted learning strategy and POS features, it performs on 50 boosting iterations, shallow trees, and 2-grams.

The POS 2-grams with the highest feature importance comprised punctuation signs followed by a conjunction (eg, ". Und," “. Aber," or ", oder"), adverbs and auxiliary verbs (eg, "dann habe/n" and "da hat/ben"), prepositions and determinative articles (eg, "mit einer/m" and "mit der/m," [ie, "with a(n)" and "with the"]) and adverbs followed by verbs (eg, "aber weisst," "mehr gegessen," and "selber gefahren").

Finally, considering word embeddings, SVM with radial basis kernel (and scaling) on augmented data outperformed all other classifiers, with $\mathrm{K}=5$ neighbors used by SMOTE ( $\mathrm{F} 1=0.44)$, followed by ADA algorithms $(\mathrm{F} 1=0.42)$ on $\mathrm{K}=9$ SMOTE neighbors. The model with SVM shows much higher recall (0.59), AUC, and AP, while the model with ADA improves precision (0.43) and consequently specificity (0.97).

\section{Discussion}

\section{Principal Results}

The primary purpose of this study was to leverage NLP features and machine learning strategies to detect reminiscence in the conversations of older adults in German in a naturalistic observation study. We used the written transcripts of the conversations and a manually coded variable (reminiscence or not reminiscence) as the basis for the prediction. We considered a wide array of methodologies, including different NLP features (ie, bag-of-words, POS tagging, and pretrained German word embeddings), multiple machine learning algorithms, and learning strategies to handle class imbalance. Results indicate that selected combinations (see below) of learning strategies, NLP features, and machine learning models show the potential to detect reminiscence. We argue that their performance can be further improved through feature engineering, by combining NLP features, using NLP-driven data augmentation techniques and collecting more data.

\section{Learning Strategies}

Class weighting SVM outperforms others machine learning models, with the highest performance seen when trained on bag-of-words $(\mathrm{F} 1=0.48)$ with a balanced precision-recall trade-off, high specificity (0.98), $\mathrm{AUC}=0.91$, and $\mathrm{AP}=0.56 . \mathrm{MC}$ strategies show lower F1 scores than class weighted and data augmentation ones, with highly imbalanced precision-recall trade-offs, resulting in low precision and, consequently, low F1 score and specificity. On the other hand, data augmentation with SMOTE delivers performance comparable to the one of class weighting, although only when using word embeddings. However, SMOTE is a purely computational approach to data augmentation, as it generates new data points from any numerical representation of transcripts (eg, on word embeddings). On the other hand, data augmentation algorithms, such as replacing with synonyms, random inserting, swapping, or deleting words [71], process transcripts directly and have improved text classification performance across multiple data sets [71]. We will further investigate the use of NLP-driven data augmentation methodologies and feature engineering [72] in forthcoming studies.

\section{Machine Learning Classifiers}

Overall, SVM delivered the highest performance across the class-weighted and data augmentation learning strategies, and boosting methodologies for the MC strategy. SVM proved to exhibit competitive performance in NLP tasks with imbalanced data sets, such as detecting offensive language (including German) [55,73,74], while the XGB algorithm proved its effectiveness in a vast array of machine learning problems [75,76]. Consequently, RF learning methods were outperformed by all learning strategies and NLP features.

\section{NLP Features}

Bag-of-words and word embeddings delivered the highest performance of all learning strategies; machine learning models trained on POS features delivered low performance due to their low precision (combined with low recall, such as in the case of ADA for class weighting and data augmentation strategies). 
Considering models trained on bag-of-words features, German stopwords related to past tense (eg, "gewesen"), personal pronouns (eg, "ich" and "wir"), and connecting words (eg, "und" and "aber") showed high feature importance, suggesting that they can encode the time, personal narrative, and structure of reminiscence. Models trained on POS tags, however, use both 1-gram and 2-grams; in particular, 2-grams comprising punctuation signs followed by a conjunction, such as ". Und" and ". Aber," suggest that reminiscing is a multisentence act during which the speaker pauses and uses forms of emphasis to elevate certain clauses to positions of more influence and importance. We will further investigate these points in future studies, introducing linguistic measures for reminiscence transcripts like other studies [24,77] to improve bag-of-words and POS models with additional sets of features to encode the phrase structure of a sentence and combining bag-of-words and POS features together.

\section{Error Analysis}

Based on an error analysis conducted on the false positives and false negatives for all the models in Table 2, the models tend to predict long transcripts with multiple sentences referring to the past incorrectly as reminiscence (ie, false positives). This results in low precision, affecting the F1 score of all classifiers. On the other hand, false negatives (ie, transcripts incorrectly classified as not reminiscence) are typically short transcripts with few words; more coded transcriptions will help to reduce both errors, in particular the false positives, enlarging the corpus of conversations and improving vocabulary richness.

In this study, we considered only transcripts in German; therefore, we have no specific insight into the specific challenges of detecting reminiscence in another language, such as English. However, earlier studies [24] have compared the application of NLP methods on transcripts of daily conversations in both German and English. Evaluated on the same set of methods and analysis pipeline, the results have suggested no significant difference in performance between the two languages. The use of different NLP features, classifiers, and learning strategies discussed in this study seems promising to develop a system for the real-time detection of reminiscence in everyday conversations in German of older adults. Such a system could leverage audio-to-text software [78] of advanced methods from automated coding [24] to automate the transcription of conversations before NLP preprocessing and the computation of machine learning predictions.

\section{Limitations}

This study has several limitations. The data set of conversations used to classify reminiscence had a limited number of records due to the short duration of the naturalistic observation study (4 days) [6]. Moreover, as we considered data from a single observation study, we cannot infer the generalizability of the presented results to other data sources. The results are based on a single partitioning of data into train and test sets. In this study, we used SpaCy pretrained German word embeddings; clearly, these embeddings are trained on corpora that may not fully encode the linguistic specificities characterizing conversations among older adults. In addition, due to the small data set available for this study, we refrained from training embeddings as well as performing machine learning with data-intensive deep learning models [79].

\section{Comparison With Prior Work}

Previous research focused on reminiscence as a therapy against dementia have shown the efficacy of information and communication technologies [14]; in particular, studies addressing the topic of automated reminiscence therapy aim at eliciting reminiscence from the users rather than detecting the presence of reminiscence in everyday life $[17,18]$. These studies have assumed that reminiscence is elicited in a setting with a human companion (eg, a therapist) or through a digital companion device using the Wizard of $\mathrm{Oz}$ technique [19]. Few studies have attempted to identify the presence of reminiscence with methods from pattern recognition and machine learning. For example, Naini et al [80] proposed a machine learning model for ranking posts from social media to create life summaries and retain memorable Facebook posts, that is, posts in a user's timeline worth remembering. A retention model based on the learning-to-rank RankSVM algorithm [81] selects posts. Alternatively, Kikhia et al [82] proposed a method to identify places of importance in lifelogging events using clustering methods and locational sensor data. The use of lifelogs allows individuals to reminisce by recalling memories, experiences, and valuable past events for fun, as a personal diary, or as a support for people with memory problems [83].

Data from Demiray et al's observational study [6] have been recently used in studies using NLP methodologies and machine learning. For example, one study [24] used their data to address the more general problem of coding social and cognitive variables from the transcripts of older adults' daily conversations. They trained multiple families of classifiers (ie, SVM, decision trees, and RF) on NLP features, including latent Dirichlet allocations and data augmentation, achieving good performance in different categories. Another study [77] used their data to compute linguistic features (ie, entropy and number of clauses) to examine the effects of age on the use of real-life language in contexts with social interactions. However, to our knowledge, this is the first study investigating the detection of reminiscence directly from the transcripts of daily conversations. Due to the presence of imbalanced data and the type of language (German) used in the conversations, this study addresses problems that are only partially present in prior scientific works in the reminiscence literature.

\section{Conclusions}

This work provides evidence to support the use of NLP and machine learning in detecting reminiscence based on written transcripts of older adults' everyday conversations in German. These results represent a novelty in the literature on reminiscence. The proposed methodology can be applied to larger natural observation studies to investigate the differences in reminiscing among cultures, countries, and social contexts using, in particular, lexical features and linguistic measures.

The aim of the WHO healthy aging model is to empower individuals to observe, measure, monitor, and take earlier action for their own health; reminiscence is an essential activity for promoting healthy aging among older adults. Real-time detection 
of reminiscence in everyday conversations can offer valuable information for older adults to understand their own health behaviors in everyday life and to support their autonomy in health maintenance.

Therefore, the training of high-performance classifiers supports the design of digital health interventions to improve older adults' quality of life by supporting their healthy aging through the real-time monitoring of reminiscence events in everyday conversations. The digital interventions may consist of a daily diary function to support the contextualization of identified reminiscing events and suggest reminiscence-related positive activities, such as replaying positive life stories and prompting more active social interactions. Another possible implementation is a conversational agent-based digital support function to support users in detecting dysfunctional reminiscence events by suggesting coping strategies. The effectiveness of the interventions could be measured by quantifying their effect on depression, quality of life, and social behavior scores, therefore assessing the impact of reminiscence therapies on healthy aging in everyday life contexts.

\section{Acknowledgments}

AF thanks Dominik Stammbach for fruitful conversations on NLP. BD thanks Marianne Mischler for the manual coding of reminiscence and reminiscence functions. KY is funded by the European Social Fund, project number ESF/14-BM-A55-0018/19. MM is partially supported by Velux Stiftung Grant 917.

\section{Authors' Contributions}

AF is the corresponding author for this work; he designed and evaluated the NLP and machine learning pipelines for this study, and drafted the manuscript. BD was responsible of the original EAR study design and data collection. BD, ML, and MM provided important intellectual inputs on reminiscence theory, the healthy aging model, and the naturalistic observational study, which helped refine the NLP pipelines. KY provided important intellectual inputs on the NLP and machine learning pipelines, as well as revising them. All authors contributed substantially to paper revisions and its finalization.

\section{Conflicts of Interest}

None declared.

\section{Multimedia Appendix 1}

Machine learning modeling: all results.

[DOCX File, 16 KB-Multimedia Appendix 1]

\section{References}

1. World report on ageing and health. World Health Organization. 2015. URL: http://www.who.int/ageing/publications/ world-report-2015/en/ [accessed 2020-03-28]

2. Martin M, Weibel R, Röcke C, Boker SM. Semantic Activity Analytics for Healthy Aging: Challenges and Opportunities. IEEE Pervasive Comput 2018 Jul;17(3):73-77. [doi: 10.1109/mprv.2018.03367738]

3. Seifert A, Christen M, Martin M. Willingness of Older Adults to Share Mobile Health Data with Researchers. GeroPsych 2018 Mar;31(1):41-49. [doi: 10.1024/1662-9647/a000181]

4. Cesari M, Araujo de Carvalho I, Amuthavalli Thiyagarajan J, Cooper C, Martin FC, Reginster J, et al. Evidence for the Domains Supporting the Construct of Intrinsic Capacity. J Gerontol A Biol Sci Med Sci 2018 Nov 10;73(12):1653-1660. [doi: 10.1093/gerona/gly011] [Medline: 29408961]

5. Butler RN. The life review: an interpretation of reminiscence in the aged. Psychiatry 1963 Feb;26:65-76. [doi: 10.1080/00332747.1963.11023339] [Medline: 14017386]

6. Demiray B, Mischler M, Martin M. Reminiscence in Everyday Conversations: A Naturalistic Observation Study of Older Adults. J Gerontol B Psychol Sci Soc Sci 2019 Jun 14;74(5):745-755. [doi: 10.1093/geronb/gbx141] [Medline: 29190392]

7. Bluck S, Levine L. Reminiscence as autobiographical memory: a catalyst for reminiscence theory development. Ageing and Society 1998 Mar 01;18(2):185-208. [doi: 10.1017/s0144686x98006862]

8. O'Rourke N, Cappeliez P, Claxton A. Functions of reminiscence and the psychological well-being of young-old and older adults over time. Aging Ment Health 2011 Mar;15(2):272-281. [doi: 10.1080/13607861003713281] [Medline: 21140308]

9. Bluck S, Alea N, Demiray B. You get what you need: The psychosocial functions of remembering. In: Mace JH, editor. New perspectives in cognitive psychology. The act of remembering: Toward an understanding of how we recall the past. Hoboken, New Jersey: Wiley-Blackwell; 2010:284-307.

10. Webster JD, Bohlmeijer ET, Westerhof GJ. Mapping the Future of Reminiscence: A Conceptual Guide for Research and Practice. Res Aging 2010 May 24;32(4):527-564. [doi: 10.1177/0164027510364122]

11. Westerhof G, Bohlmeijer E, Webster J. Reminiscence and mental health: a review of recent progress in theory, research and interventions. Ageing and Society 2010 Mar 01;30(4):697-721. [doi: 10.1017/s0144686x09990328] 
12. Pinquart M, Forstmeier S. Effects of reminiscence interventions on psychosocial outcomes: a meta-analysis. Aging Ment Health 2012;16(5):541-558. [doi: 10.1080/13607863.2011.651434] [Medline: 22304736]

13. Webster JD, Cappeliez P. Reminiscence and Autobiographical Memory: Complementary Contexts for Cognitive Aging Research. Developmental Review 1993 Mar;13(1):54-91. [doi: 10.1006/drev.1993.1003]

14. Lazar A, Thompson H, Demiris G. A systematic review of the use of technology for reminiscence therapy. Health Educ Behav 2014 Oct;41(1 Suppl):51S-61S [FREE Full text] [doi: 10.1177/1090198114537067] [Medline: 25274711]

15. Schwarz N. Why researchers should think "real-time": A cognitive rationale. In: Mehl MR, Conner TS, editors. Handbook of research methods for studying daily life. US: The Guilford Press; 2012:22-42.

16. Bielak AAM. How can we not 'lose it' if we still don't understand how to 'use it'? Unanswered questions about the influence of activity participation on cognitive performance in older age--a mini-review. Gerontology 2010;56(5):507-519 [FREE Full text] [doi: 10.1159/000264918] [Medline: 19996570$]$

17. Caros M, Garolera M, Radeva P, Giro-i-Nieto X. Automatic Reminiscence Therapy for Dementia. ArXiv. URL: http://arxiv. org/abs/1910.11949 [accessed 2019-10-25]

18. Nikitina S, Callaioli S, Baez M. Smart conversational agents for reminiscence. In: SE4COG '18: Proceedings of the 1st International Workshop on Software Engineering for Cognitive Services. New York, NY, USA: Association for Computing Machinery; 2018 Presented at: ICSE '18: 40th International Conference on Software Engineering; May 2018; Gothenburg Sweden p. 52-57. [doi: 10.1145/3195555.3195567]

19. Vardoulakis L, Ring L, Barry B, Sidner C, Bickmore T. Designing Relational Agents as Long Term Social Companions for Older Adults. In: Nakano Y, Neff M, Paiva A, Walker M, editors. IVA'12: Proceedings of the 12th international conference on Intelligent Virtual Agents. Berlin, Heidelberg: Springer; 2012:289-302.

20. Demiray B, Luo M, Tejeda-Padron A, Mehl MR. Sounds of Healthy Aging: Assessing Everyday Social and Cognitive Activity from Ecologically Sampled Ambient Audio Data. In: Hill P, Allemand M, editors. Personal Healthy Aging Adulthood New Dir Tech. Cham: Personal Healthy Aging Adulthood New Dir Tech Internet; 2020:111-132.

21. Woods B, O'Philbin L, Farrell EM, Spector AE, Orrell M. Reminiscence therapy for dementia. Cochrane Database Syst Rev 2018 Mar 01;3:CD001120 [FREE Full text] [doi: 10.1002/14651858.CD001120.pub3] [Medline: 29493789]

22. Subramaniam $P$, Woods B. The impact of individual reminiscence therapy for people with dementia: systematic review. Expert Rev Neurother 2012 May;12(5):545-555. [doi: 10.1586/ern.12.35] [Medline: 22550983]

23. Seyfert-Margolis V. The evidence gap. Nat Biotechnol 2018 Mar 06;36(3):228-232. [doi: 10.1038/nbt.4097] [Medline: 29509737]

24. Yordanova K, Demiray B, Mehl MR, Martin M. Automatic Detection of Everyday Social Behaviours and Environments from Verbatim Transcripts of Daily Conversations. In: Proc IEEE Int Conf Pervasive Comput Commun. Proc IEEE Int Conf Pervasive Comput Commun Internet Kyoto, Japan: IEEE; 2019 Presented at: Pervasive Computing and Communications (PerCom), 2019 IEEE International Conference on; March; Kyoto, Japan p. 242-251. [doi: 10.1109/percom.2019.8767403]

25. Liew J, McCracken N, Zhou S, Crowston K. Optimizing Features in Active Machine Learning for Complex Qualitative Content Analysis. USA: Association for Computational Linguistics; 2014 Presented at: Proc ACL 2014 Workshop Lang Technol Comput Soc Sci; June; Baltimore, MD, USA p. 44-48. [doi: 10.3115/v1/w14-2513]

26. Colneric N, Demsar J. Emotion Recognition on Twitter: Comparative Study and Training a Unison Model. IEEE Trans. Affective Comput 2020 Jul 1;11(3):433-446. [doi: 10.1109/taffc.2018.2807817]

27. Joshi A, Sparks R, McHugh J, Karimi S, Paris C, MacIntyre CR. Harnessing Tweets for Early Detection of an Acute Disease Event. Epidemiology 2020;31(1):90-97. [doi: 10.1097/ede.0000000000001133]

28. Alburez-Gutierrez D, Zagheni E, Aref S, Gil-Clavel S, Grow A, Negraia D. Demography in the Digital Era: New Data Sources for Population Research. SocArXiv. 2019. URL: https://osf.io/preprints/socarxiv/24jp7/

29. Funk B, Sadeh-Sharvit S, Fitzsimmons-Craft EE, Trockel MT, Monterubio GE, Goel NJ, et al. A Framework for Applying Natural Language Processing in Digital Health Interventions. J Med Internet Res 2020 Feb 19;22(2):e13855 [FREE Full text] [doi: 10.2196/13855] [Medline: $\underline{\text { 32130118] }}$

30. Cappeliez P, O'Rourke N. Empirical validation of a model of reminiscence and health in later life. J Gerontol B Psychol Sci Soc Sci 2006 Jul 01;61(4):P237-P244. [doi: 10.1093/geronb/61.4.p237] [Medline: 16855036]

31. Korte J, Bohlmeijer ET, Westerhof GJ, Pot AM, Pot AM. Reminiscence and adaptation to critical life events in older adults with mild to moderate depressive symptoms. Aging Ment Health 2011 Jul 01;15(5):638-646. [doi: 10.1080/13607863.2010.551338] [Medline: 21815856]

32. O'Rourke N, King DB, Cappeliez P. Reminiscence functions over time: consistency of self functions and variation of prosocial functions. Memory 2016 May 04;25(3):403-411. [doi: 10.1080/09658211.2016.1179331] [Medline: 27145425]

33. Boker SM, Brick TR, Pritikin JN, Wang Y, von Oertzen T, Brown D, et al. Maintained Individual Data Distributed Likelihood Estimation (MIDDLE). Multivariate Behav Res 2015;50(6):706-720 [FREE Full text] [doi: 10.1080/00273171.2015.1094387] [Medline: 26717128]

34. Al-Rubaie M, Chang JM. Privacy-Preserving Machine Learning: Threats and Solutions. IEEE Secur Privacy 2019 Mar;17(2):49-58. [doi: 10.1109/msec.2018.2888775] 
35. Mehl MR, Pennebaker JW, Crow DM, Dabbs J, Price JH. The Electronically Activated Recorder (EAR): a device for sampling naturalistic daily activities and conversations. Behav Res Methods Instrum Comput 2001 Nov;33(4):517-523. [doi: $10.3758 / \mathrm{bf03195410}$ ] [Medline: $\underline{11816455]}$

36. Folstein MF, Folstein SE, McHugh PR. "Mini-mental state": A practical method for grading the cognitive state of patients for the clinician. J Psychiatr Res 1975 Nov;12(3):189-198. [doi: 10.1016/0022-3956(75)90026-6] [Medline: 1202204]

37. Mehl MR. The Electronically Activated Recorder (EAR): A Method for the Naturalistic Observation of Daily Social Behavior. Curr Dir Psychol Sci 2017 Apr;26(2):184-190 [FREE Full text] [doi: 10.1177/0963721416680611] [Medline: 28529411]

38. Jurafsky D, Martin JH. Speech and Language Processing (2nd edition). Upper Saddle River, NJ, USA: Prentice-Hall, Inc; 2009.

39. Manning C, Schütze H. Foundations of Statistical Natural Language Processing. Cambridge, MA: MIT Press; 1999.

40. Chen J, Lalor J, Liu W, Druhl E, Granillo E, Vimalananda VG, et al. Detecting Hypoglycemia Incidents Reported in Patients' Secure Messages: Using Cost-Sensitive Learning and Oversampling to Reduce Data Imbalance. J Med Internet Res 2019 Mar 11;21(3):e11990 [FREE Full text] [doi: 10.2196/11990] [Medline: 30855231]

41. Leroy G, Gu Y, Pettygrove S, Galindo MK, Arora A, Kurzius-Spencer M. Automated Extraction of Diagnostic Criteria From Electronic Health Records for Autism Spectrum Disorders: Development, Evaluation, and Application. J Med Internet Res 2018 Nov 07;20(11):e10497 [FREE Full text] [doi: 10.2196/10497] [Medline: 30404767]

42. Guetterman TC, Chang T, DeJonckheere M, Basu T, Scruggs E, Vydiswaran VV. Augmenting Qualitative Text Analysis with Natural Language Processing: Methodological Study. J Med Internet Res 2018 Jun 29;20(6):e231 [FREE Full text] [doi: 10.2196/jmir.9702] [Medline: 29959110]

43. Pedregosa F, Varoquaux G, Gramfort A, Michel V, Thirion B, Grisel O, et al. Scikit-learn: Machine Learning in Python. J Mach Learn Res 2011;12:2825-2830 [FREE Full text]

44. spaCy | Industrial-strength Natural Language Processing in Python. URL: https://spacy.io/ [accessed 2020-04-06]

45. Harris ZS. Distributional Structure. WORD 1954 Aug;10(2-3):146-162. [doi: 10.1080/00437956.1954.11659520]

46. German | spaCy Models Documentation. URL: https://spacy.io/models/de [accessed 2020-04-06]

47. Brants S, Dipper S, Eisenberg P, Hansen-Schirra S, König E, Lezius W, et al. TIGER: Linguistic Interpretation of a German Corpus. Res Lang Comput 2004 Dec 1;2(4):597-620. [doi: 10.1007/s11168-004-7431-3]

48. Nachrichten aus Deutschland und der Welt | Frankfurter Rundschau. URL: https://www.fr.de/ [accessed 2020-04-06]

49. Nothman J, Ringland N, Radford W, Murphy T, Curran JR. Learning multilingual named entity recognition from Wikipedia. Artificial Intelligence 2013 Jan 01;194:151-175. [doi: 10.1016/j.artint.2012.03.006]

50. Fernández A, García S, Galar M, Prati R, Krawczyk B, Herrera F. Learning from Imbalanced Data Sets 1st edition. New York, NY: Springer; 2018.

51. He H, Ma Y. Imbalanced Learning: Foundations, Algorithms, and Applications, 1st edition. Hoboken, New Jersey: Wiley-IEEE Press; 2013.

52. Krawczyk B. Learning from imbalanced data: open challenges and future directions. Prog Artif Intell 2016 Apr 22;5(4):221-232. [doi: 10.1007/s13748-016-0094-0]

53. López V, Fernández A, García S, Palade V, Herrera F. An insight into classification with imbalanced data: Empirical results and current trends on using data intrinsic characteristics. Information Sciences 2013 Nov 20;250:113-141. [doi: 10.1016/j.ins.2013.07.007]

54. Padurariu C, Breaban ME. Dealing with Data Imbalance in Text Classification. Procedia Computer Science 2019 Jan 01;159:736-745. [doi: 10.1016/j.procs.2019.09.229]

55. GermEval 2018 - Germeval Task 2, 2019 — Shared Task on the Identification of Offensive Language. URL: https://projects. fzai.h-da.de/iggsa/germeval-2018/ [accessed 2020-04-06]

56. Chawla NV, Bowyer KW, Hall LO, Kegelmeyer WP. SMOTE: Synthetic Minority Over-sampling Technique. jair 2002 Jun 01;16:321-357. [doi: 10.1613/jair.953]

57. Hastie T, Tibshirani R. The Elements of Statistical Learning: Data Mining, Inference, and Prediction, Second Edition. New York, NY, USA: Springer Series in Statistics Springer New York Inc; 2001.

58. Chen T, Guestrin C. XGBoost: A Scalable Tree Boosting System. 2016 Presented at: KDD '16: The 22nd ACM SIGKDD International Conference on Knowledge Discovery and Data Mining; 2016; San Francisco California USA p. $785-794$. [doi: $10.1145 / 2939672.2939785]$

59. Fawcett T. An introduction to ROC analysis. Pattern Recognition Letters 2006 Jun 01;27(8):861-874. [doi: 10.1016/j.patrec.2005.10.010]

60. Jin Huang, Ling C. Using AUC and accuracy in evaluating learning algorithms. IEEE Trans. Knowl. Data Eng 2005 Mar;17(3):299-310. [doi: 10.1109/TKDE.2005.50]

61. sklearn.metrics.average_precision_score — scikit-learn 0.22.2 documentation. URL: https://scikit-learn.org/stable/modules/ generated/sklearn.metrics.average precision score.html [accessed 2020-04-06]

62. Saito T, Rehmsmeier M. The precision-recall plot is more informative than the ROC plot when evaluating binary classifiers on imbalanced datasets. PLoS One 2015 Mar 04;10(3):e0118432 [FREE Full text] [doi: 10.1371/journal.pone.0118432] [Medline: $\underline{25738806]}$ 
63. López V, Fernández A, Moreno-Torres JG, Herrera F. Analysis of preprocessing vs. cost-sensitive learning for imbalanced classification. Open problems on intrinsic data characteristics. Expert Systems with Applications 2012 Jun;39(7):6585-6608. [doi: 10.1016/j.eswa.2011.12.043]

64. Wang H, Cui Z, Chen Y, Avidan M, Abdallah AB, Kronzer A. Predicting Hospital Readmission via Cost-Sensitive Deep Learning. IEEE/ACM Trans Comput Biol Bioinform 2018 Dec;15(6):1968-1978. [doi: 10.1109/TCBB.2018.2827029] [Medline: 29993930]

65. sklearn.ensemble.RandomForestClassifier — scikit-learn 0.22.2 documentation. URL: https://scikit-learn.org/stable/modules/ generated/sklearn.ensemble.RandomForestClassifier.html [accessed 2020-04-06]

66. XGBoost Parameters — xgboost 1.1.0 documentation. URL: https://xgboost.readthedocs.io/en/latest/parameter.html [accessed 2020-04-06]

67. Rokach L. Ensemble-based classifiers. Artif Intell Rev 2009 Nov 19;33(1-2):1-39. [doi: 10.1007/s10462-009-9124-7]

68. Siers M, Islam M. Software defect prediction using a cost sensitive decision forest and voting, and a potential solution to the class imbalance problem. Information Systems 2015 Jul;51:62-71 [FREE Full text] [doi: 10.1016/j.is.2015.02.006]

69. Ramezankhani A, Pournik O, Shahrabi J, Azizi F, Hadaegh F, Khalili D. The Impact of Oversampling with SMOTE on the Performance of 3 Classifiers in Prediction of Type 2 Diabetes. Med Decis Making 2016 Jan;36(1):137-144. [doi: 10.1177/0272989X14560647] [Medline: 25449060]

70. Blagus R, Lusa L. SMOTE for high-dimensional class-imbalanced data. BMC Bioinformatics 2013 Mar 22;14(1):106 [FREE Full text] [doi: 10.1186/1471-2105-14-106] [Medline: 23522326]

71. Wei J, Zou K. EDA: Easy Data Augmentation Techniques for Boosting Performance on Text Classification Tasks. In: Proceedings of the 2019 Conference on Empirical Methods in Natural Language Processing and the 9th International Joint Conference on Natural Language Processing (EMNLP-IJCNLP).: Association for Computational Linguistics; 2019 Presented at: EMNLP | IJCNLP; November 2019; Hong Kong, China p. 6382-6388 URL: https://www.aclweb.org/anthology/D19-1670/ [doi: $10.18653 / \mathrm{v} 1 / \mathrm{d} 19-1670]$

72. Nobata C, Tetreault J, Thomas A, Mehdad Y, Chang Y. Abusive Language Detection in Online User Content. In: Proc 25th Int Conf World Wide Web. 2016 Presented at: WWW '16: 25th International World Wide Web Conference; April; Montréal, Québec, Canada. [doi: 10.1145/2872427.2883062]

73. Schmidt A, Wiegand M. A Survey on Hate Speech Detection using Natural Language Processing. In: Proceedings of the Fifth International Workshop on Natural Language Processing for Social Media. Proc Fifth Int Workshop Nat Lang Process Soc Media Internet Valencia, Spain: Association for Computational Linguistics; 2017 Presented at: SocialNLP|WS; April; Valencia, Spain p. 1-10. [doi: 10.18653/v1/w17-1101]

74. Wiegand M, Ruppenhofer J, Schmidt A, Greenberg C. Inducing a Lexicon of Abusive Words - a Feature-Based Approach. In: Proceedings of the 2018 Conference of the North American Chapter of the Association for Computational Linguistics: Human Language Technologies, Volume 1 (Long Papers).: Association for Computational Linguistics; 2018 Presented at: NAACL; June 2018; New Orleans, Louisiana p. 1046-1056 URL: https://www.aclweb.org/anthology/N18-1095/ [doi: $10.18653 / \mathrm{v} 1 / \mathrm{n} 18-1095]$

75. The Present and the Future of the KDD Cup Competition: an Outsider's Perspective. URL: https://www.linkedin.com/pulse/ present-future-kdd-cup-competition-outsiders-ron-bekkerman/ [accessed 2020-04-06]

76. Ferrario A, Hämmerli R. On Boosting: Theory and Applications. Rochester, NY: Social Science Research Network; 2019. URL: https://papers.ssrn.com/abstract=3402687 [accessed 2020-04-06]

77. Luo M, Schneider G, Martin M, Demiray B. Cognitive Aging Effects on Language Use in Real-Life Contexts: A Naturalistic Observation Study. In: University of Zurich. 2019 Presented at: 41st Annual Conference of the Cognitive Science Society; July 24-27, 2019; Montreal URL: https://www.zora.uzh.ch/id/eprint/174992/ [doi: 10.5167/uzh-174992]

78. Ziman K, Heusser AC, Fitzpatrick PC, Field CE, Manning JR. Is automatic speech-to-text transcription ready for use in psychological experiments? Behav Res Methods 2018 Dec 23;50(6):2597-2605. [doi: 10.3758/s13428-018-1037-4] [Medline: 29687235]

79. Goodfellow I, Bengio Y, Courville A. Deep Learning. Cambridge, MA: MIT Press; 2016.

80. Naini KD, Kawase R, Kanhabua N, Niederée C, Altingovde IS. Those were the days: learning to rank social media posts for reminiscence. Inf Retrieval J 2018 Aug 11;22(1-2):159-187. [doi: 10.1007/s10791-018-9339-9]

81. Joachims T. Optimizing search engines using clickthrough data. In: Proc Eighth ACM SIGKDD Int Conf Knowl Discov Data Min. Edmonton, Alberta, Canada: Association for Computing Machinery; 2002 Presented at: KDD02: The Eighth ACM SIGKDD International Conference on Knowledge Discovery and Data Mining; July; Edmonton, Alberta, Canada p. 133-142. [doi: 10.1145/775047.775067]

82. Kikhia B, Boytsov A, Hallberg J, ul Hussain Sani Z, Jonsson H, Synnes K. Structuring and Presenting Lifelogs Based on Location Data. In: Cipresso P, Matic A, Lopez G, editors. Pervasive Computing Paradigms for Mental Health. Cham: Springer International Publishing; 2014:133-144.

83. Kikhia B, Hallberg J, Bengtsson JE, Savenstedt S, Synnes K. Building digital life stories for memory support. IJCIH 2010;1(2):161. [doi: 10.1504/ijcih.2010.037460] 


\author{
Abbreviations \\ ADA: adaptive boosting \\ AP: average precision \\ AUC: area under the curve \\ CLW: class-weighted learning \\ EAR: Electronically Activated Recorder \\ NLP: natural language processing \\ POS: part-of-speech \\ RF: random forests \\ SMOTE: Synthetic Minority Oversampling Technique \\ SVM: support vector machines \\ tf-idf: term frequency-inverse document frequency \\ WHO: World Health Organization \\ XGB: extreme gradient boosting
}

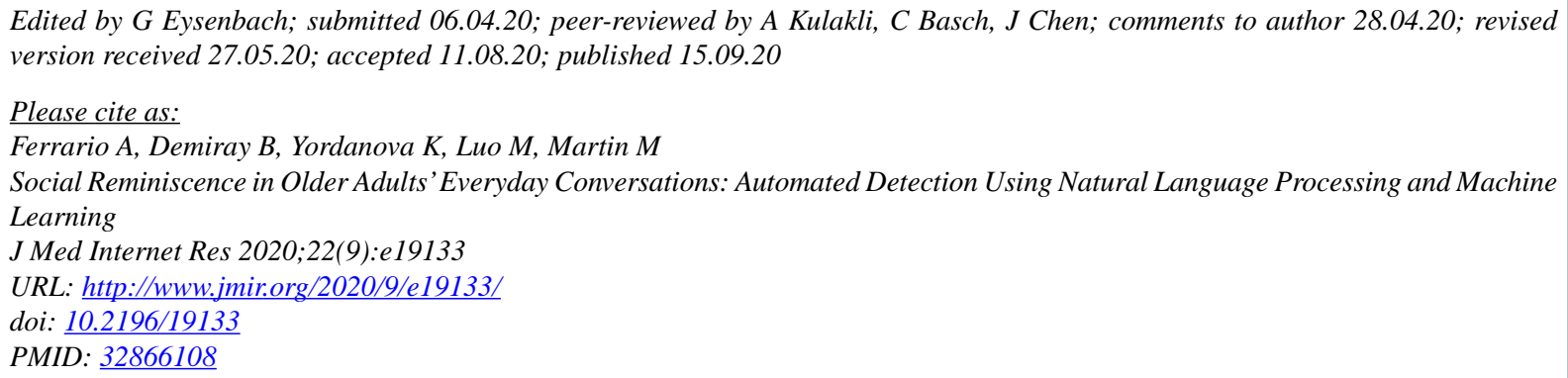

(C) Andrea Ferrario, Burcu Demiray, Kristina Yordanova, Minxia Luo, Mike Martin. Originally published in the Journal of Medical Internet Research (http://www.jmir.org), 15.09.2020. This is an open-access article distributed under the terms of the Creative Commons Attribution License (https://creativecommons.org/licenses/by/4.0/), which permits unrestricted use, distribution, and reproduction in any medium, provided the original work, first published in the Journal of Medical Internet Research, is properly cited. The complete bibliographic information, a link to the original publication on http://www.jmir.org/, as well as this copyright and license information must be included. 